\title{
SEMIPRIMENESS OF SPECIAL JORDAN ALGEBRAS
}

\author{
KEVIN MCCRIMMON ${ }^{1}$
}

\begin{abstract}
There are important connections between radicals of a special Jordan algebra $J$ and its associative envelope $A$. For the locally nilpotent (Levitzki) radical $\mathscr{L}$, Skosyrskii proved $\mathscr{L}(J)=J \cap \mathscr{L}(A)$. For the prime (Baer) radical $\mathscr{P}$, Erickson and Montgomery proved $\mathscr{P}(J)=J \cap \mathscr{P}(A)$ when $J=H(A, *)$ consists of all symmetric elements of an algebra $A$ with involution *. In his important work on prime Jordan algebras, Zelmanov proved $\mathscr{P}(J)=J \cap \mathscr{P}(A)$ for all linear $J$ and all associative envelopes $A$. In the present paper we extend Zelmanov's result to arbitrary quadratic Jordan algebras. In particular, we see that a special Jordan algebra is semiprime iff it has some semiprime associative envelope.
\end{abstract}

Throughout this paper we work with algebras over an arbitrary ring of scalars $\Phi$. A special Jordan algebra is a subspace $J \subset A$ of an associative algebra $A$ closed under the Jordan products

$$
x^{2}=x x \text { and } U_{x} y=x y x
$$

and hence under their linearizations

$$
x \circ y=x y+y x \text { and }\{x y z\}=x y z+z y x .
$$

Note that in linear Jordan algebras, where $\frac{1}{2} \in \Phi$, we can recover $U_{x} y$ from $x^{2}$ since

$$
2 U_{x} y=x \circ(x \circ y)-x^{2} \circ y \text {. }
$$

As usual, the commutator or Lie product in $A$ is denoted by $[x, y]=x y-y x$. Since all our Jordan algebras live inside associative algebras, we do not need the axioms or identities characterizing abstract Jordan algebras-all identitites we use are immediate consequences of the above definitions of Jordan products. We do not assume our algebras are unital, and on occasion it will be notationally convenient to use the unital hulls $\hat{A}=\Phi 1 \oplus A, \hat{J}=\Phi 1 \oplus J$ obtained by formally adjoining a unit.

An associative algebra $A$ is semiprime if it has no nonzero nilpotent ideals $B \triangleleft A$ ( $B^{n}=0$ for some $n$, where $B^{1}=B$ and $B^{n+1}=B B^{n}$, for $B C$ the linear span of all elements $b c$ for $b \in B, c \in C)$, equivalently no trivial ideals $\left(B^{2}=0\right)$, equivalently no nonzero trivial elements $z(z A z=0)$. There is a unique ideal $P \triangleleft A$ minimal among those whose quotient is semiprime; this prime or Baer radical $\mathscr{P}(A)=P$ may be recursively constructed as $\mathscr{P}(A)=\mathscr{P}_{\lambda_{0}}(A)$ for suitably large ordinal $\lambda_{0}$, where $\mathscr{P}_{0}(A)=0, \mathscr{P}_{\lambda+1}(A)=\sum\left\{B \mid B \triangleleft A\right.$ is nilpotent modulo $\left.\mathscr{P}_{\lambda}(A)\right\}$, and $\mathscr{P}_{\lambda}(A)$ $=\bigcup_{\mu<\lambda} \mathscr{P}_{\mu}(A)$ for a limit ordinal $\lambda$.

Received by the editors June $28,1984$.

1980 Mathematics Subject Classification. Primary 16A68; Secondary 17C20, 17C50.

Key words and phrases. Special Jordan algebra, semiprime, associative enveloping algebra.

${ }^{1}$ Research partially supported by NSF Grant MCS-82-02103.

(C) 1986 American Mathematical Society $0002-9939 / 86 \$ 1.00+\$ .25$ per page 
An ideal $I \triangleleft J$ in a Jordan algebra is a subspace $I \subset J$ such that any Jordan product in $J$ having a factor from $I$ falls back in $I$ (this may be succinctly phrased as $\left.U_{I} \hat{J}+U_{\hat{J}} I \subset I\right)$. A Jordan algebra $J$ is semiprime if it has no nilpotent ideals $I \triangleleft J\left(I^{[n]}=0\right.$ for some $n$, where the Jordan power $I^{[n]}$ consists of all Jordan products from $I$ of degree $\geqslant n$, counting $U_{x} y$ as degree 2 in $x$; here $I^{[n]} \subset I^{n}$ when $J \subset A$ ), equivalently no solvable ideals (some $I^{(n)}=0$, where $I^{(0)}=I$ and $I^{(n+1)}=$ $U_{I^{(n)}} I^{(n)}$; here $I^{(n)}$ remains an ideal in $J$ if $I$ is, in contrast to $I^{[n]}$; when $J \subset A$ we have $\left.I^{(n)} \subset I^{3^{n}}\right)$, equivalently no trivial ideals $\left(I^{(1)}=U_{I} I=I^{[3]}=0\right)$. ( $J$ is strongly semiprime if it has no trivial elements $U_{z} J=0$; in general this is more restrictive than just being semiprime.) Again there is a unique ideal $\mathscr{P}(J) \triangleleft J$ minimal among those with semiprime quotient, and it may be recursively constructed as $\mathscr{P}_{\lambda}(J)$ as in the associative case via $\mathscr{P}_{\lambda+1}(J)=\sum\left\{I \mid I \triangleleft J\right.$ is nilpotent modulo $\left.\mathscr{P}_{\lambda}(J)\right\}$. (We obtain a slightly quicker chain of $\tilde{\mathscr{P}}_{\lambda}(J)$ by summing the $I$ which are solvable.)

We say an associative $A$ is an associative envelope for $J \subset A$ if $J$ generates $A$ as associative algebra.

Semiprime Envelope Theorem [3, Lemma 6, p. 93]. If $J$ is a special Jordan algebra, then

$$
\mathscr{P}(J)=J \cap \mathscr{P}(A)
$$

for any associative envelope $A$ for $J$. Thus a special Jordan algebra is semiprime iff it has some semiprime associative envelope.

Proof. We begin by showing that

$$
\mathscr{P}(A) \cap J \subset \mathscr{P}(J) .
$$

This will show that if $J$ is semiprime then it has a semiprime associative envelope $\bar{A}=A / \mathscr{P}(A) \quad(J \cap \mathscr{P}(A) \subset \mathscr{P}(J)=0$ implies $\quad J=J / J \cap \mathscr{P}(A) \simeq J+$ $\mathscr{P}(A) / \mathscr{P}(A) \subset A / \mathscr{P}(A))$. In view of the recursive construction of $\mathscr{P}(A)$, it suffices to establish

$$
\mathscr{P}_{\lambda}(A) \cap J \subset \mathscr{P}_{\lambda}(J)
$$

by induction on $\lambda$. The case $\lambda=0$ is trivial $\left(\mathscr{P}_{0}(A)=0\right)$, as is the case of a limit ordinal $\lambda\left(\mathscr{P}_{\lambda}(A)=\bigcup_{\mu<\lambda} \mathscr{P}_{\mu}(A)\right.$ where $\mathscr{P}_{\mu}(A) \cap J \subset \mathscr{P}_{\mu}(J)$ by the induction hypothesis), so we need only prove the assertion $\left(1^{\prime}\right)$ for $\lambda+1$ given the assertion for $\lambda$. Now $\mathscr{P}_{\lambda+1}(A)$ is the sum of all ideals $B \triangleleft A$ which are nilpotent modulo $\mathscr{P}_{\lambda}(A)$, $B^{n} \subset \mathscr{P}_{\lambda}(A)$ for some $n=n(B)$. We claim we have an elemental characterization

$$
\mathscr{P}_{\lambda+1}(A)=\left\{x \in A \mid \hat{A} x \hat{A} \text { is nilpotent modulo } \mathscr{P}_{\lambda}(A)\right\} \text {. }
$$

Certainly $\mathscr{P}_{\lambda+1}(A)$ contains all such $x$, and conversely any $x \in \mathscr{P}_{\lambda+1}(A)$ is such an element: $x$ has the form $x=x_{1}+\cdots+x_{r}$ for $x_{k} \in B_{k} \triangleleft A, B_{k}^{n_{k}} \subset \mathscr{P}_{\lambda}(A)$, so if we set $n=n(x)=\left(n_{1}-1\right)+\cdots+\left(n_{r}-1\right)+1$ then $(\hat{A} x \hat{A})^{n}=\sum \hat{A} x_{i_{1}} \hat{A} x_{i_{2}} \cdots \hat{A} x_{i_{n}} \hat{A}$ where in each summand some $x_{k}$ appears at least $n_{k}$ times (else each appears at most $n_{k}-1$ times, and there would be at most $\left(n_{1}-1\right)+\cdots+\left(n_{r}-1\right)=n-1$ total factors $\left.x_{k}\right)$, and therefore falls in $B_{k}^{n_{k}} \subset \mathscr{P}_{\lambda}(A)$, so $(\hat{A} x \hat{A})^{n} \subset \mathscr{P}_{\lambda}(A)$. This establishes (2). 
To prove $\left(1^{\prime}\right)$ for $\lambda+1$, if $x \in \mathscr{P}_{\lambda+1}(A) \cap J$ then the Jordan ideal $I(x) \triangleleft J$ generated by $x$ has $I(x) \subset \hat{A} x \hat{A}$, so by (2) there is an $n$ such that $I(x)^{[n]} \subset$ $(\hat{A} x \hat{A})^{n} \cap J \subset \mathscr{P}_{\lambda}(A) \cap J \subset \mathscr{P}_{\lambda}(J)$ by the induction hypothesis on $\lambda$; by definition of $\mathscr{P}_{\lambda+1}(J)$ we have $I(x) \subset \mathscr{P}_{\lambda+1}(J)$, therefore $x \in \mathscr{P}_{\lambda+1}(J)$. This completes the inductive proof of $\left(1^{\prime}\right)$, hence establishes the inclusion (1). This direction does not use that $A$ is an envelope, only that $A \supset J$.

The reverse inclusion

$$
\mathscr{P}(J) \subset J \cap \mathscr{P}(A)
$$

is more difficult, at least for quadratic algebras where $\frac{1}{2} \notin \Phi$. It can be reformulated as saying that if $J$ has a semiprime envelope it must itself be semiprime,

$$
A \text { semiprime } \Rightarrow J \text { semiprime }
$$

(clearly (3) $\Rightarrow\left(3^{\prime}\right)$ since semiprimeness means $\mathscr{P}=0$, and conversely $\left(3^{\prime}\right) \Rightarrow(3)$ since $\bar{A}=A / \mathscr{P}(A)$ is a semiprime envelope for $\bar{J}=J+\mathscr{P}(A) / \mathscr{P}(A) \simeq J / J \cap \mathscr{P}(A)$, and $\bar{J}$ semiprime forces $\mathscr{P}(J) \subset J \cap \mathscr{P}(A)$ by minimality of $\mathscr{P}(J)$ ). Since semiprimeness of $J$ amounts to absence of trivial ideals, $\left(3^{\prime}\right)$ is equivalent to

$$
A \text { semiprime, } \quad I^{[3]}=0 \text { for } I \triangleleft J \Rightarrow I=0 .
$$

This is the version we will establish. From now on we assume $A$ is a semiprime envelope for $J$ and $I$ is a trivial ideal in $J$, and we will prove that $I=0$.

We begin by developing some criteria for things to vanish in a semiprime envelope. First we have

CRITERION I. If $S \subset A$ is a subspace with $J S \subset S \hat{A}$ (equivalently, $J \circ S \subset S \hat{A}$ or $[J, S] \subset S \hat{A})$, then

$$
z S=0(z \in S \hat{A}) \Rightarrow z=0,
$$

since $J S \subset S \hat{A}$ for the generating set $J$ shows $A S \hat{A} \subset S \hat{A}$ and hence $z A z \subset z A S \hat{A} \subset$ $z S \hat{A}=0$ forces $z=0$ by semiprimeness of $A$. When $K \triangleleft J$ is a Jordan ideal we have $J \circ K \subset K$, so Criterion I for $S=K$ becomes

Criterion II. If $K \triangleleft J$ is a Jordan ideal then

$$
z K=0(z \in K \hat{A}) \Rightarrow z=0 .
$$

When $c$ commutes with $J$ and has square zero, $c^{2}=[J, c]=0$, then $c(\Phi c)=[J, \Phi c]$ $=0$, so Criterion I for $S=\Phi c$ becomes

Criterion III. If $c \in A$ has $c^{2}=[c, J]=0$ then $c=0$. (Alternately, observe $c$ is nilpotent in the center of $A$ when it commutes with the generators $J$, hence $c=0$ by semiprimeness.) Combining the last two special cases gives

CRITERIOn IV. If $K \triangleleft J$ is an ideal then

$$
U_{z} K=\{z \hat{J} K\}=0(z \in K \hat{A}) \Rightarrow z=0,
$$

since by Criterion II it suffices to show $z K=0$, i.e. $c=z w=0$ for all $w \in K$, and this follows from Criterion III since $c^{2}=z w z w=0$ if $U_{z} K=0$, and $[c, J]=0$ if $\{z \hat{J} K\}=0$ since for all $x \in J$ we have $[c, x]=z w x-x z w=z w x+x w z-$ $x\{z 1 w\}=\{z, 1, w \circ x\}-\{z x w\}-x\{z 1 w\} \in \hat{J}\{z \hat{J} K\}=0$. 
Criterion $\mathrm{I}$ is in turn the special case $K=\hat{J}$ of a more general

Criterion V. If $S \subset K \hat{A}$ is a subspace with $J S K \subset S \hat{A}$ for some ideal $K \triangleleft J$, then

$$
z S=0(z \in S \hat{A}) \Rightarrow z=0
$$

since then $z \in S^{\prime}=\{w \in S \hat{A} \mid w S=0\}$, where $S^{\prime} J S=0$ by Criterion II $\left(S^{\prime} J S \subset K \hat{A}\right.$ has $\left(S^{\prime} J S\right) K=S^{\prime}(J S K) \subset S^{\prime}(S \hat{A})=\left(S^{\prime} S\right) \hat{A}=0$ by hypothesis on $S$ and definition of $\left.S^{\prime}\right)$, thus $S^{\prime} J \subset S^{\prime}$ shows $S^{\prime} A \subset S^{\prime}$, and therefore $z A z \subset\left(S^{\prime}\right) A(S \hat{A}) \subset S^{\prime} S \hat{A}$ $=0$ forces $z=0$ by semiprimeness of $A$.

Using these criteria we quickly see our trivial ideal $I$ vanishes when $A$ has no 2-torsion. Denote by $I^{\langle 2\rangle}$ the span of all Jordan squares $z^{2}$ for $z \in I$, thus including all $z \circ w$ (not the associative square $I^{2}=I I$ spanned by all associative products $z w$ ). Then $S=I^{\langle 2\rangle}$ and $K=I$ satisfy the hypotheses of Criterion $\mathrm{V}\left(J I^{\langle 2\rangle} I \subset I^{\langle 2\rangle} \hat{A}\right.$ when $I^{[3]}=0$ since for $x \in J, z, w \in I$ we have $x z^{2} w=\{x \circ z, z, w\}-U_{z} x \circ w-$ $\{w z z\} x+z^{2} w x \in I^{[3]}-I \circ I-I^{[3]} J+I^{\langle 2\rangle} A \subset I^{\langle 2\rangle} \hat{A}$ ), and $S S=0$ (by $z^{2} w^{2}=$ $\{z z w\} w-w z^{2} w \in I^{[3]} \hat{J}=0$ ), so by Criterion $\mathrm{V}$ we have $S=0$ :

$$
I^{\langle 2\rangle}=0 .
$$

Applying Criterion IV to $K=I$ and $z \in 2 I$ (note $U_{z} K \subset I^{[3]}=0$ and $\{z \hat{J} K\} \subset$ $2 U_{I, I} \hat{J} \subset I \circ(I \circ \hat{J})-(I \circ I) \circ \hat{J}=0$ since $I \circ I=0$ by (4)), we see $z=0$ :

$$
2 I=0 .
$$

For linear Jordan algebras where $\frac{1}{2} \in \Phi$, this yields the desired condition $I=0$. For the general quadratic case we must argue at greater length. Since the product $U_{K} L$ of two ideals is again an ideal, we get a smaller ideal

$$
I^{\prime}=U_{I} \hat{J} \triangleleft J, \quad I^{\prime} \subset I .
$$

This new ideal satisfies

$$
I^{\prime} \circ \hat{J}^{\langle 2\rangle}=0
$$

since for $z \in I, \quad x, y \in \hat{J}$ we have $U_{z} y \circ x^{2}=z \circ\left\{y z x^{2}\right\}-U_{z} x^{2} \circ y \in I \circ I-$ $\left\{(z \circ x)^{2}-U_{x} z^{2}-z \circ U_{x} z\right\} \circ y \subset I \circ I-\left\{I^{\langle 2\rangle}-U_{\hat{J}} I^{\langle 2\rangle}-I \circ I\right\} \circ \hat{J}=0$ by (4). In view of (5) this shows $I^{\prime}$ commutes with $J^{\langle 2\rangle}$; we claim it actually commutes with $J$,

$$
\left[I^{\prime}, J\right]=0 \text {, }
$$

i.e. $\left[x, I^{\prime}\right]=0$ for each $x$ in $J$. Now $S=\left[x, I^{\prime}\right]=x \circ I^{\prime}$ (via (5)) satisfies the hypotheses of Criterion I:

$$
\begin{aligned}
J \circ S & =J \circ\left(x \circ I^{\prime}\right)=2 U_{J, x} I^{\prime}-x \circ\left(J \circ I^{\prime}\right)+(J \circ x) \circ I^{\prime} \subset 0-x \circ I^{\prime}+0 \\
& =S
\end{aligned}
$$

by (5), $I^{\prime} \triangleleft J$, and (6). Furthermore, $S S=0$ since for $z^{\prime}, w^{\prime} \in I^{\prime}$ we have

$$
\begin{gathered}
\left(x \circ z^{\prime}\right)\left(x \circ w^{\prime}\right)=x z^{\prime}\left(x \circ w^{\prime}\right)+z^{\prime} x\left(x \circ w^{\prime}\right) \\
=-x\left(x \circ w^{\prime}\right) z^{\prime}+z^{\prime} x^{2} w^{\prime}+z^{\prime} x w^{\prime} x \\
\quad\left(z^{\prime} \in I \text { anticommutes with } x \circ w^{\prime} \in I\right. \text { by (4)) } \\
=-x^{2} w^{\prime} z^{\prime}-x w^{\prime} x z^{\prime}-x^{2} z^{\prime} w^{\prime}-z^{\prime} x w^{\prime} x+2 z^{\prime} x w^{\prime} x \\
\quad\left(z^{\prime} \in I^{\prime} \text { anticommutes with } x^{2}\right. \text { by (6)) } \\
=-x^{2}\left(w^{\prime} \circ z^{\prime}\right)-\left(x w^{\prime} x\right) \circ z^{\prime}+2 z^{\prime} x w^{\prime} x \\
=0 \quad(\text { by (4), (5)). }
\end{gathered}
$$


Thus by Criterion I we have $S=0,\left[x, I^{\prime}\right]=0$ for each $x$, and (7) holds. But then we can apply Criterion III to any $c \in I^{\prime}$ (note $c^{2} \in I^{\langle 2\rangle}=0$ by (4)) to see $c=0$, thus $I^{\prime}$ vanishes:

$$
I^{\prime}=\dot{0}
$$

In particular, $\{I \hat{J} I\} \subset I^{\prime}=0$, so by Criterion IV for $K=I$ we see any $z \in I$ vanishes,

$$
I=0
$$

as desired. This completes the proof of $\left(3^{\prime \prime}\right)$ and the Theorem.

\section{REFERENCES}

1. T. S. Erickson and M. S. Montgomery, The prime radical in special Jordan rings, Trans. Amer. Math. Soc. 156 (1971), 155-164.

2. V. G. Skosyrskiī, On nilpotence in Jordan and right alternative algebras, Algebra i Logika 18 (1979), 73-85.

3. E. I. Zelmanov, On prime Jordan algebras. II, Sibirsk Mat. J. 24 (1983), 89-104.

Department of Mathematics, University of Virginia, Charlottesville, Virginia 22903 